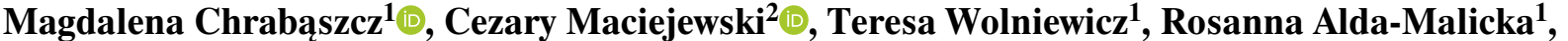 Patrycja Gajda $^{1}$, Joanna Czuwara ${ }^{1}{ }^{(}$, Lidia Rudnicka ${ }^{1}(\mathbb{D}$
}

${ }^{1}$ Department of Dermatology, Medical University of Warsaw, Poland

${ }^{2} 1^{\text {st }}$ Department of Cardiology, Medical University of Warsaw, Poland

\section{Access to a dermatoscope during dermatology courses motivates students' towards thorough skin examination}

\author{
Address for correspondence: \\ Joanna Czuwara MD, PhD \\ Department of Dermatology \\ Medical University of Warsaw, Poland \\ Koszykowa 82A, 02-008 Warszawa \\ e-mail: jczuwara@gmail.com
}

Oncology in Clinical Practice

2021, Vol. 17, No. 2, 47-52

DOI: $10.5603 /$ OCP. 2020.0040

Copyright $(C) 2021$ Via Medica

ISSN 2450-1654

e-ISSN: 2450-6478

\begin{abstract}
Introduction. Dermatoscope is a tool for a skin examination, used especially in early detection of malignant skin lesions. Non-dermatologists are being trained for opportunistic melanoma detection with the usage of dermatoscopy, however, still non-satisfactory. This study was aimed to determine whether practical dermoscopy adjunct to traditional, lecture and seminar-based medical school curriculum would improve the perceived relevance of regular skin examination and basic skin lesions differentiation.

Material and method. Fourth-year medical students participating in a 3-week-long dermatology course were randomly assigned to two groups: the first one called $A$ with limited access to a dermatoscope and the second one called B, with unlimited access to dermatoscopes throughout the course. All participants answered surveys concerning their attitude towards skin examination, with a rating scale from 1 to 5 , before and after the course. Also, all participants completed an image-based dermoscopy test for distinguishing benign from malignant skin lesions. Results. Students assigned to group B significantly improved their perceived importance of routine skin examination (mean scores before 4.38; after 4.57, P = 0.03). No such tendency was observed in group $A$ - before 4.40, after $4.49(P=0.29)$. Students in the group with higher dermatoscope availability considered buying a dermatoscope more often (61\%) than those without $(44 \%)(P=0.037)$. No significant score difference was observed when testing skin lesions identification, mean for extended access 7.84 vs. normal 7.64 points $(P=0.69)$.

Conclusions. Higher availability of dermatoscopy during dermatology rotations may encourage students to use this tool in future clinical practice and improve early detection of malignant skin lesions.

Key words: dermatoscopy, medical education, skin cancer, melanoma, skin exam
\end{abstract}

Oncol Clin Pract 2021; 17, 2: 47-52

\section{Introduction}

The incidence of melanoma and nonmelanoma skin cancers continues to increase [1]. Melanoma is the cause of the majority of skin cancer deaths, but the keratinocyte-based malignancies of basal cell carcinoma and squamous cell carcinoma also have an important impact on the public health burden $[2,3]$.

Melanoma survival exceeds $90 \%$ for 5-year relative rate in Nordic or Western countries, but is below $60 \%$ in Eastern Europe for people diagnosed with melanoma as recently as this decade [4]. Mortality due to melanoma is
$20 \%$ higher in Poland than the average for the European Union (2010), with the main reason being late diagnosis of the disease, already in an advanced stage [5, 6].

Several meta-analyses have identified risk factors for skin cancer occurrence, such as intermittent sun exposure (especially at a young age), a history of sunburn, positive family history, light skin phenotype, the density of freckles, blond hair, pre-malignant skin lesions and actinic damage indicators [7-9]. Most cases are attributable to modifiable risk factors, by far the most significant being exposure to ultraviolet radiation [10]. Early diagnosis remains the best method to reduce 
melanoma and non-melanoma skin cancer-related mortality and morbidity [11].

Dermoscopy is a non-invasive technique that consists of viewing pigmented skin lesions through a hand-held lens. It has evolved over 20 years and is now widely used for dermatological examination. According to studies, this technique increases the sensitivity and specificity for the diagnosis of early melanoma in comparison to examination with the unaided eye. This simple procedure increases melanoma diagnostic accuracy by up to $35 \%$ [12-14]. Dermatoscopic examination is currently a standard procedure for clinical differential diagnosis of cutaneous melanoma and recommendation for excisional biopsy $[6,15]$. Early diagnosis of cutaneous melanoma is crucial to ensure the timely excision of thin mostly curable lesions. It can also lead to the detection of thinner and smaller cancers and it results in a cost-effective cancer screening strategy [16]. Surgical removal of cutaneous melanoma not only improves prognosis but is also associated with approximately $90 \%$ likelihood of cure [17].

The introduction of pocket-size and handy dermoscopes could significantly lead to the broader use of dermatoscopy. However, as with any clinical tool alike, training is required for the effective use [18]. Non-dermatologist physicians are well-positioned for opportunistic melanoma detection, yet education regarding skin cancer examination is limited during medical school, and practical skills are poorly acquired by mostly lecture-based teaching. Workshop-based teaching is far more effective, especially where practical skills are concerned. Additionally, teaching methods that encourage self-directed learning can be effective in delivering core knowledge, leading to an enhanced level of self-education [19-22].

Firstly, the purpose of this study was to determine whether increased dermatoscope availability during the standard fourth-year dermatology course improves the ability of medical students to distinguish benign, suspicious and malignant lesions. Secondly, the study aimed to investigate students' perspectives on the role of skin examination and determine whether a simple intervention may increase its importance in medical students' opinion.

\section{Material and method}

A three-week dermatology course is part of the fourth-year medical school curriculum at Medical University in Warsaw. The course is divided into a clinical part where students take medical history and perform a physical examination on patients in small, 4-people subgroups and a lecture part that discusses common skin diseases. The intensive seminar-based introduction to dermatoscopic lesion identification/differentiation is also a relevant part of the students' course.
The current study was performed prospectively in 2 cohorts of fourth-year medical students at the Department of Dermatology of our university.

Student groups were randomly assigned to:

- cohort A (10 groups) with standard, limited access to dermatoscopes under close supervision from their teaching physician only - approximately 1 dermatoscope per 15 people;

- cohort B (10 groups) with unlimited access to dermatoscopes throughout the course - 1 dermatoscope per 4-person subgroup plus a short tutorial covering technical aspects of dermatoscopic examination using the device.

After 3-week long dermatology course, students in both study arms completed an image-based test of 12 lesions (Fig. 1) to evaluate the gained knowledge and experience in differentiation between malignant and benign skin lesions.

Additionally, at the beginning and the end of the course, students answered a short survey that covered topics such as previous experience with the field of dermatology, attitudes towards skin examination, and perceived importance of skin examination by non-dermatologists.

\section{Statistical analysis}

Wilcoxon signed-rank test was used for comparison of scores: (1) perceived importance of routine skin examination in the pre- and post-intervention survey within cohorts A and B; (2) scores in the 12-skin lesions image based test in cohort A and B; (3) scores in the 12-skin lesions image based test according to dermatology as planned specialty, planned surgical specialty, student gender, family history of skin cancer, skin disorders in family history; (4) scores in the self-assessment of dermatoscopy skills in cohort A and B.

Fisher's exact test was used for comparison of proportions of students considering dermatoscope possession in the future between cohort A and B.

\section{Results}

\section{Group characteristics}

Overall, there were 230 responses from before and 156 responses after the dermatology course. Median student age was 22 years and $57 \%$ were women. $9.13 \%$ of participants had a case of skin cancer in their close family members, $30 \%$ admitted the presence of any skin disease in their closest family. 30.1\% had undergone at least one dermatoscopy examination in the past. $12.2 \%$ considered dermatology as a future specialty. According to participants, only $3.3 \%$ had an opportunity to attend a course or workshop related to dermatoscopy before the dermatology 

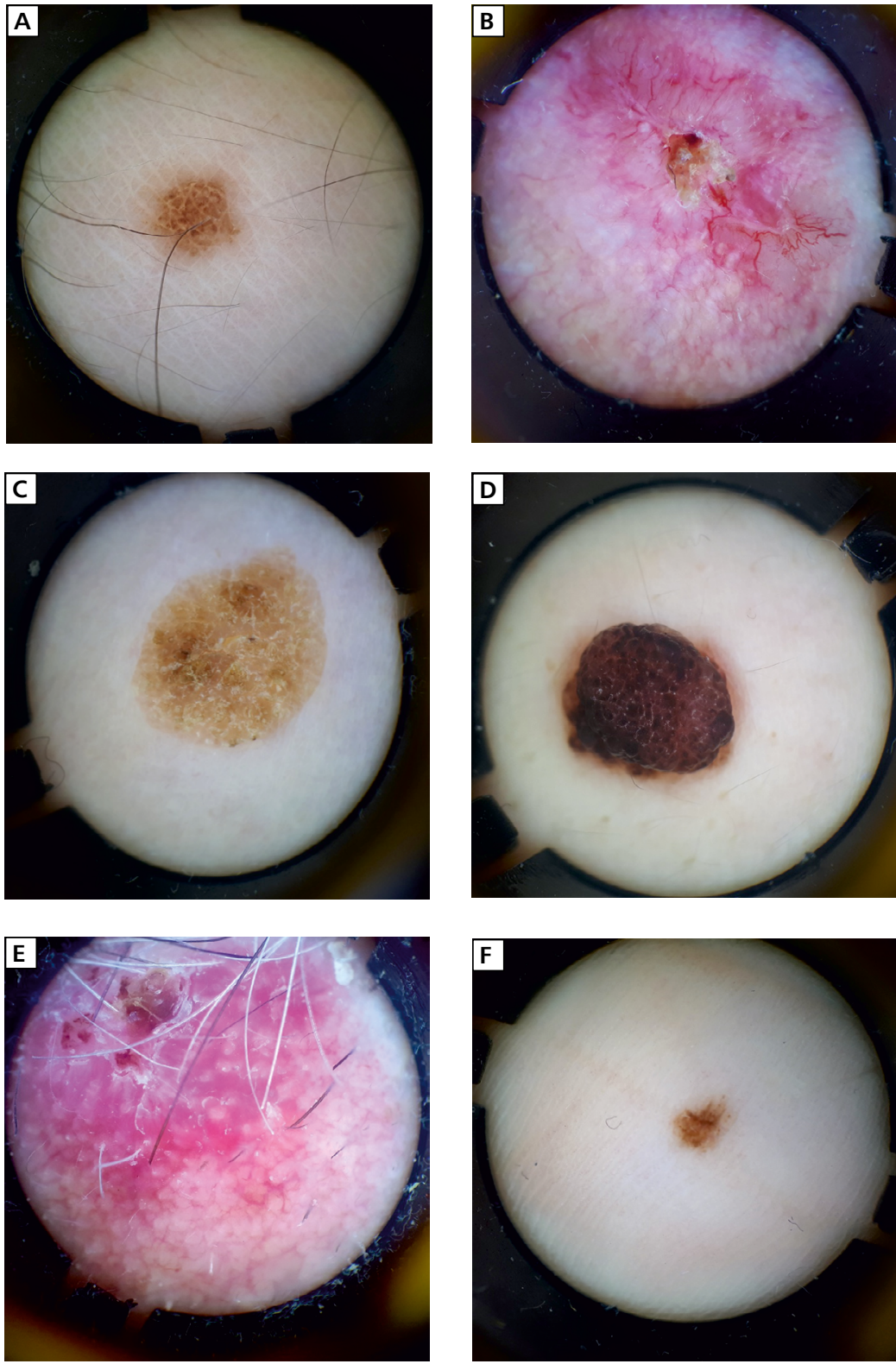

Figure 1. Examples of dermoscopy pictures used in the image-based test. The pictures were performed via the handy model of dermatoscope, the same model that students used during classes; A. Nevus; B. Basal cell carcinoma; C. Seborrheic keratosis; D. Congenital nevus; E. Actinic keratosis; F. Acral nevus

course. $55.6 \%$ of the study population were assigned to the groups with extended dermatoscopes availability (cohort B) and the rest (44.4\%) consisted of cohort A - with usual limited dermatoscopy access during the course. Of those available in follow-up, $52.9 \%$ were in cohort B.

\section{Results}

Students assigned to cohort B - with full access to dermatoscopes - significantly improved their perceived importance of routine skin examination (mean score before -4.38 , after $-4.57, \mathrm{P}=0.03$ ) (Fig. 2). There was no such tendency in cohort A - without extended access to dermatoscopes (before - 4.40, after - 4.49, $\mathrm{P}=0.29)$. Students in cohort $\mathrm{B}$ considered buying a dermatoscope in the future more often $(61 \%)$ than those in cohort A $(44 \%)(\mathrm{P}=0.037)$.

There were no significant differences in results of the final test on recognition of skin lesions - mean score in cohort $\mathrm{B}-7.84$ points $v s .7 .64$ in cohort $\mathrm{A}(\mathrm{P}=0.69)$.

There were no significant differences in the self-assessment of dermatoscopy skills in both groups (ranked using a 5-point scale) - mean result 2.99 in cohort B vs. 2.88 in cohort $\mathrm{A}(\mathrm{P}=0.32)$. 


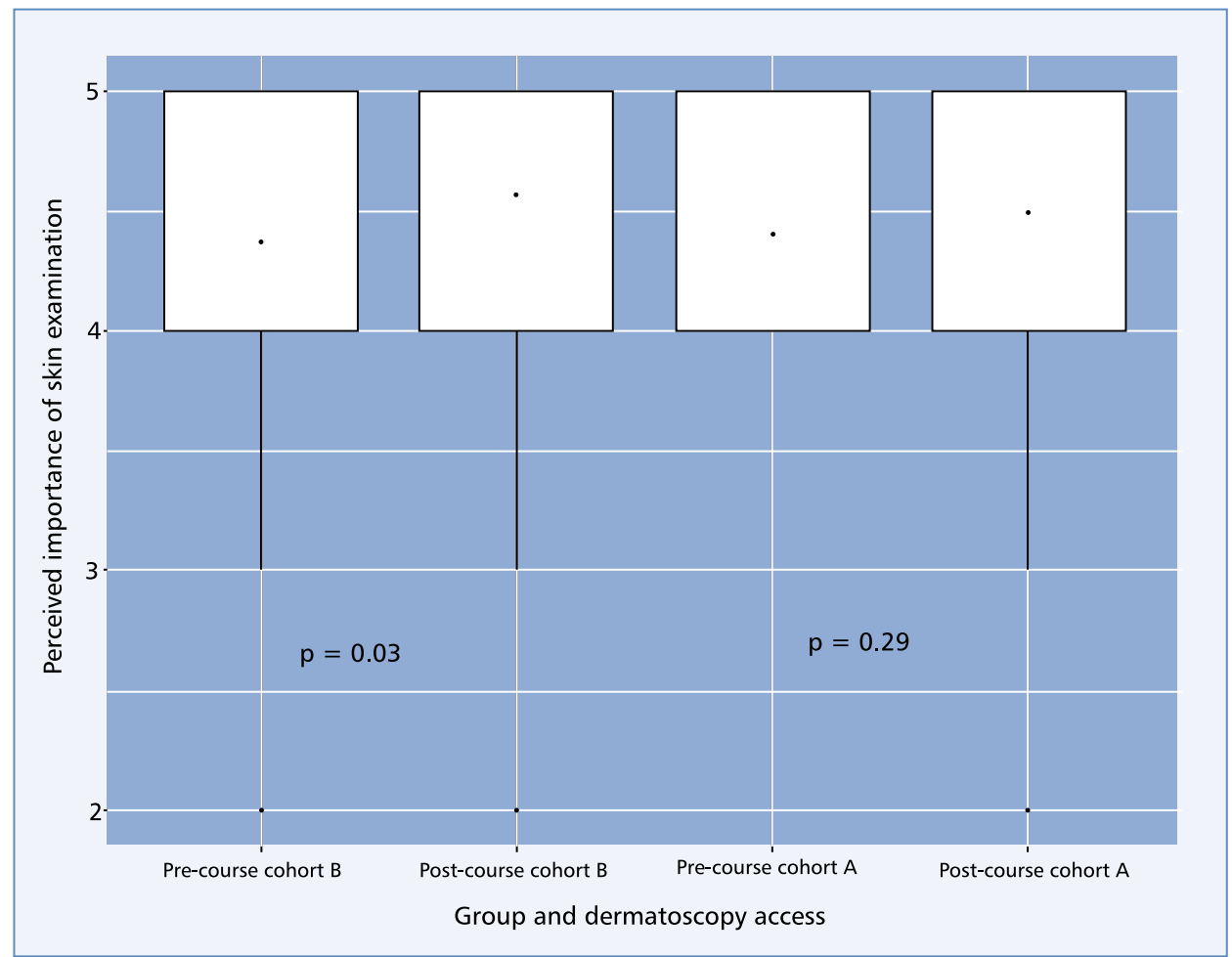

Figure 2. Changes in perceived importance of routine skin examination in cohort $A$ and cohort B. P-values refer to change within respective students' cohort (group B - with dermatoscope access; group A - without dermatoscope access)

Interestingly, dermatology as a planned specialty (dermatology as an intended specialty -7.79 , other field as planned specialty $-7.74, \mathrm{P}=0.97)$, planned surgical specialty (planned surgical specialty -7.82 , other specialty planned $-7.55, \mathrm{P}=0.38$ ), female sex (females -7.64 , males $-7.92, \mathrm{P}=0.60)$, history of skin cancer in closest family members (positive history for skin cancer -7.63 , negative history for skin cancer -7.77 , $\mathrm{P}=0.90)$, and presence of a skin disorder in the closest family (positive history of skin disorders - 7.70, negative history of skin disorders $-7.71, \mathrm{P}=0.70$ ) were also not associated with better skin lesion recognition skills.

\section{Discussion and conclusion}

This study demonstrates that the adjunct of dermoscopy to the standard fourth-year medical curriculum could boost students' awareness of the importance of performing careful skin examination.

Education in medical schools regarding the diagnosis of cutaneous lesions is minimal, and students are exposed to very little training and practice in dermato$\operatorname{logy}[23]$.

The lack of practical teaching of dermatoscopy almost without a doubt reduces the likelihood that newly graduating physicians will include thorough examina- tions for skin cancer in their routine practice. Most patients with melanoma were observed to visit a general practitioner within a year before diagnosis. Therefore, many melanomas could potentially be detected earlier if physicians were better prepared and educated in melanoma identification and paid closer attention to the skin examination [24]. Considering this, intervention through encouraging skin examination and personal dermatoscope ownership may prove beneficial in the long-term as these students might be more inclined to develop their skills and use dermatoscopy in their future everyday clinical practice.

According to the participants, only $3.3 \%$ had an opportunity to attend a course or workshop (including theoretical) related to dermatoscopy before their dermatology course. The observation that medical students receive very little training or practice in skin cancer detection is consistent with previously reported insufficient performance by non-dermatologist physicians and doctors-in-training in skin cancer screening. Apart from the introduction of practical dermatoscopy training during dermatology classes, initiation of dermatoscopic lesion differentiation in preclinical subjects (e.g. as part of pathology courses) could increase the awareness of the importance of skin evaluation in future physicians.

In our study students who trained with dermatoscopy readily available did not exhibit greater skills in 
distinguishing melanoma from benign neoplasms when compared with students who had classes with limited access to the tool. This may be due to the fact that the standard course of dermatology for the $4^{\text {th }}$ year students was simply too short to produce a substantial difference in knowledge.

In a study performed by Tracey et al. students receiving access to dermoscopy improved in the diagnosis of cutaneous lesions compared with those not receiving the dermoscopy intervention. However, in this study, the skin cancer examination intervention lasted 2 consecutive years. Additionally, the group with dermatoscopes received a dermoscopy tutorial [20]. The intervention in the present study was based solely on the improvement of dermatoscope availability during the course for some of the students.

Another reason might be that the ability to confidently diagnose benign and malignant skin lesions with a dermatoscope is gained with time and practice. Previous observations show, that during the training and learning phase, clinicians tend to increase their sensitivity but have lower specificity of their dermatoscopic diagnoses. In fact, during the first year after learning dermoscopy, generally the benign to malignant ratio increases. However, after gaining further experience, clinicians specificity also increases, resulting in improved benign to malignant ratios compared with an examination via the naked eye [25]. The phenomenon described above might partially explain why the students with the increased availability of dermatoscopy in practice did not show better results in the final quiz.

Interestingly, in a similar piece of research conducted by Chen et al. [26] medical students receiving a skin cancer examination intervention with the aid of dermoscopy improved their diagnostic skills more significantly after a period of one year. In this study cohort 1 received skin cancer examination teaching only and cohort 2 received skin cancer examination teaching with dermoscopy tutorial, and a dermatoscope.

Both cohorts improved or maintained their scores to correctly classify all lesions from post-intervention to one-year follow-up. After one year, cohort 2 maintained higher scores for successful identification of both benign and malignant lesions in comparison with cohort 1 [26].

The present study has a few limitations. Firstly, participants in the clinical assessment reflect students from a single institution, which limits generalizability. Secondly, differences seen in interventions A versus B may result from differences in the university setting (e.g. academic teachers, group interests and course outline) that students were exposed to during the course. However, no change other than the introduction of dermatoscopes in randomly selected groups of students was implemented during the study. Thirdly, not all participants filled in the postintervention survey as completion of the survey was optional. Nonetheless, response rates for both cohorts were approximately equal. In addition, the data does not show the long- term impact of our intervention. Follow-up would be necessary in order to establish whether students maintained a long-term interest in skin examination and dermatoscopy. Finally, the 12 samples of skin lesions used for the image-based test might not have been enough to objectively capture the students' knowledge or clinical skill level.

This study illustrates the need and shows the benefits, of adding practical dermatoscopy education to the standard medical school dermatology curriculum. Providing even a small opportunity for practical training with dermatoscopy in detecting potentially fatal and aggressive skin cancers may help to increase awareness and learning regarding skin cancer detection with dermatoscopy use. The study demonstrates that access to a dermatoscope can be executed effectively even when constrained by limited curriculum time.

There is a need to optimize education related to skin cancer examination. Efforts should be made to fill medical students' competency gap in dermatoscopy that influences secondary prevention efforts [27]. General practitioners are expected to have at least basic skills in dermatology. However, primary care physicians perform skin cancer prevention or screening activities rarely and less frequently than many other screening and prevention activities. It should be acknowledged that even with the use of a dermatoscope not all lesions will be diagnosed correctly. Nevertheless, dermatoscopy appears to be a cost-effective diagnostic intervention in primary care $[28,29]$.

\section{Acknowledgments}

This research was funded by the Polish Ministry of Science and Higher Education, grant number MNiSW/2020/223/DIR/NN4.

\section{Conflict of interest}

The authors report no conflicts of interest.

\section{References}

1. Rogers HW, Weinstock MA, Feldman SR, et al. ncidence estimate of nonmelanoma skin cancer (keratinocyte carcinomas) in the U.S. population, 2012. JAMA Dermatol. 2015; 151(10): 1081-1086, doi: 10.1001/jamadermatol.2015.1187.

2. Siegel RL, Miller KD, Jemal A. Cancer statistics, 2018. CA Cancer J Clin. 2018; 68(1): 7-30, doi: 10.3322/caac.21442, indexed in Pubmed: 29313949.

3. Bickers D, Lim H, Margolis D, et al. The burden of skin diseases: 2004 a joint project of the American Academy of Dermatology Association and the Society for Investigative Dermatology. J Am Acad Dermatol. 2006; 55(3): 490-500, doi: 10.1016/j.jaad.2006.05.048. 
4. Allemani C, Matsuda T, Di Carlo V, et al. Global surveillance of trends in cancer survival 2000-14 (CONCORD-3): analysis of individual records for 37513025 patients diagnosed with one of 18 cancers from 322 population-based registries in 71 countries. Lancet. 2018; 391(10125): 1023-1075, doi: 10.1016/S0140-6736(17)33326-3.

5. Forsea AM. Melanoma epidemiology and early detection in Europe: diversity and disparities. Dermatol Pract Concept. 2020; 10(3): e2020033, doi: 10.5826/dpc. 1003a33, indexed in Pubmed: 32642304

6. Rutkowski $P$, et al., Cutaneous melanoma - diagnostic and therapeutic guidelines in 2016. Cutaneous melanoma - diagnostic and therapeutic guidelines in 2016. Oncology in Clin Pract 2015; 11(4)

7. Gandini S, Sera F, Cattaruzza MS, et al. Meta-analysis of risk factors for cutaneous melanoma: I. Common and atypical naevi. Eur J Cancer. 2005; 41(1): 28-44, doi: 10.1016/j.ejca.2004.10.015, indexed in Pubmed: 15617989

8. Gandini S, Sera F, Cattaruzza MS, et al. Meta-analysis of risk factors for cutaneous melanoma: II. Sun exposure. Eur J Cancer. 2005; 41(1): 45-60, doi: 10.1016/i.ejca.2004.10.016, indexed in Pubmed: 15617990

9. Gandini S, Sera F, Cattaruzza MS, et al. Meta-analysis of risk factors for cutaneous melanoma: III. Family history, actinic damage and phenotypic factors. Eur J Cancer. 2005; 41(14): 2040-2059, doi: 10.1016/i ejca.2005.03.034, indexed in Pubmed: 16125929

10. Brown KF, Rumgay H, Dunlop $\mathrm{C}$, et al. The fraction of cancer attributable to modifiable risk factors in England, Wales, Scotland, Northern Ireland, and the United Kingdom in 2015. Br J Cancer. 2018; 118(8) 1130-1141, doi: 10.1038/s41416-018-0029-6, indexed in Pubmed 29567982

11. Mayer JE, Swetter SM, Fu T, et al. Screening, early detection, education, and trends for melanoma: current status (2007-2013) and future directions: Part I. Epidemiology, high-risk groups, clinical strategies, and diagnostic technology. J Am Acad Dermatol. 2014; 71(4): 599. e1-599.e12; quiz 610, 599.e12, doi: 10.1016/j.jaad.2014.05.046 indexed in Pubmed: 25219716.

12. Gulia A, Brunasso AG, Massone C. Dermoscopy: distinguishing malignant tumors from benign. Expert Review of Dermatology. 2014; 7(5): 439-458, doi: 10.1586/edm.12.47.

13. Vestergaard ME Macaskill P. Holt PE et al. Dermoscopy compared with naked eye examination for the diagnosis of primary melanoma: a meta-analysis of studies performed in a clinical setting. Br J Dermatol. 2008; 159(3): 669-676, doi: 10.1111/j.1365-2133.2008.08713.x indexed in Pubmed: 18616769.

14. Bafounta ML, Beauchet A, Aegerter P, et al. Is dermoscopy (epiluminescence microscopy) useful for the diagnosis of melanoma? Results of a meta-analysis using techniques adapted to the evaluation of diagnostic tests. Arch Dermatol. 2001; 137(10): 1343-1350, doi 10.1001/archderm.137.10.1343, indexed in Pubmed: 11594860

15. Kittler $\mathrm{H}$, Pehamberger $\mathrm{H}$, Wolff $\mathrm{K}$, et al. Diagnostic accuracy of dermoscopy. Lancet Oncol. 2002; 3(3): 159-165, doi: 10.1016/s14702045(02)00679-4, indexed in Pubmed: 11902502

16. Thomas L, Puig S. Dermoscopy, digital dermoscopy and other diagnostic tools in the early detection of melanoma and follow-up of high-risk skin cancer patients. Acta Derm Venereol. 2017; Suppl 218 14-21, doi: 10.2340/00015555-2719, indexed in Pubmed: 28676882.
17. Marghoob A, Swindle L, Moricz C, et al. Instruments and new technologies for the in vivo diagnosis of melanoma. J Am Acad Dermatol. 2003; 49(5): 777-797, doi: 10.1016/s0190-9622(03)02470-8.

18. Kardynal A, Olszewska M. Modern non-invasive diagnostic techniques in the detection of early cutaneous melanoma. J Dermatol Case Rep. 2014; 8(1): 1-8, doi: 10.3315/jdcr.2014.1161, indexed in Pubmed: 24748903.

19. Marghoob AA, Usatine RP, Jaimes N. Dermoscopy for the family physician. Am Fam Physician. 2013; 88(7): 441-450

20. Liebman TN, Goulart JM, Soriano R, et al. Effect of dermoscopy education on the ability of medical students to detect skin cancer. Arch Dermatol. 2012; 148(9): 1016-1022, doi: 10.1001/archdermatol.2012.509, indexed in Pubmed: 22986850.

21. Robinson JK, Jain N Marghoob AA et al A randomized trial on the efficacy of mastery learning for primary care provider melanoma opportunistic screening skills and practice. J Gen Intern Med. 2018; 33(6): 855-862, doi: 10.1007/s11606-018-4311-3, indexed in Pubmed: 29404948.

22. Marchetti MA, Fonseca M, Dusza SW, et al. Dermatoscopic imaging of skin lesions by high school students: a cross-sectional pilot study. Dermatol Pract Concept. 2015; 5(1): 11-28, doi: 10.5826/dpc.0501a02, indexed in Pubmed: 25692076

23. Geller AC, Venna S, Prout M, et al. Should the skin cancer examination be taught in medical school? Arch Dermatol. 2002; 138(9): 1201-1203, doi: 10.1001/archderm.138.9.1201, indexed in Pubmed: 12224981.

24. Dolan NC. Skin cancer control in the primary care setting: are we making any progress? J Gen Intern Med. 2001; 16(5): 342-343, doi: 10.1046/j.1525-1497.2001.10334.x, indexed in Pubmed: 11359555.

25. Terushkin V, Warycha M, Levy M, et al. Analysis of the benign to malignant ratio of lesions biopsied by a general dermatologist before and after the adoption of dermoscopy. Arch Dermatol. 2010; 146(3): 343-344, doi: 10.1001/archdermatol.2010.12, indexed in Pubmed: 20231514.

26. Chen LL, Liebman TN, Soriano RP, et al. One-year follow-up of dermoscopy education on the ability of medical students to detect skin cancer. Dermatology. 2013; 226(3): 267-273, doi: 10.1159/000350571, indexed in Pubmed: 23797047.

27. Garg A, Wang J, Reddy SB, et al. Integrated skin exam consortium. Curricular factors associated with medical students' practice of the skin cancer examination: an educational enhancement initiative by the integrated skin exam consortium. JAMA Dermatol. 2014; 150(8): 850-855, doi: 10.1001/jamadermatol.2013.8723, indexed in Pubmed: 24898482

28. Koelink CJL, Vermeulen KM, Kollen BJ, et al. Diagnostic accuracy and cost-effectiveness of dermoscopy in primary care: a cluster randomized clinical trial. J Eur Acad Dermatol Venereol. 2014; 28(11): 1442-1449, doi: 10.1111/jdv.12306, indexed in Pubmed: 25493316.

29. Westerhoff K, McCarthy WH, Menzies SW. Increase in the sensitivity for melanoma diagnosis by primary care physicians using skin surface microscopy. Br J Dermatol. 2000; 143(5): 1016-1020, doi: 10.1046/j.1365-2133.2000.03836.x, indexed in Pubmed: 11069512. 